alternative, and the best of these enable relatively inexperienced staff to obtain accurate results. They are suitable for urgent or infrequently requested assays, but they are too expensive and time-consuming for testing large numbers of samples. The Department concluded, therefore, that the best solution lay in expanding existing laboratory facilities within the N.H.S.

The working parties on steroid and protein assays proposed a multi-tier system based on supraregional, regional, and area centres, with the intention that such a system could provide a wide range of assays while ensuring that most laboratories retained some complex manual procedures. They recommended that each area health authority should support one or more laboratories to carry out some of the common specialized assays, such as those for cortisol, digoxin, urinary pregnancy and non-pregnancy oestrogens, human placental lactogen, and thyroxine. They further proposed that one or more area centres in each region should be designated a regional centre and perform a more extensive range of assays, including those for which a large population is required to ensure adequate throughput and for which a week's delay in reporting results is acceptable. Examples include assays for progesterone, and for luteinizing, follicle stimulating, thyroid-stimulating, and growth hormones. The area and regional centres would require additional funding from the area and regional health authorities respectively, while clinicians would get the local services they needed.

The supraregional tier of the assay service has recently been established, with central funding from the Department of Health, and is based on 12 existing laboratories with expertise in the analyses required. An accurate and rapid analytical service is now available to all clinicians via the chemical pathologist or biochemist in their own hospital. More than 30 assays are offered, including those for several steroids (progesterone, testosterone, 17- $\beta$ oestradiol, aldosterone, pregnanediol, and prenanetriol) and many other hormones (including ACTH, antidiuretic hormone, calcitonin, parathyroid hormone, prolactin, gastrin, and tri-iodothyronine). The scope of this service has been extended outside the field of endocrinology to include other haptens (cyclic AMP, vitamin $B_{12}$, vitamin $\mathrm{D}$, and digoxin) and proteins ( $\alpha$-fetoprotein, carcinoembryonic antigen, and ferritin). Pathology laboratories throughout England and Wales now have a booklet listing the assays that are available and summarizing the clinical indications for such assays and how samples should be taken and transported.

The directors of these supraregional centres join representatives from other bodies in regular meetings to discuss modifications and improvements in the analytical service and how best the supraregional service can fulfil its other roles, which include the training of biochemists and technicians for regional and area centres; the supply, in conjunction with outside bodies such as the Division of Biological Standards, of antisera and labelled and unlabelled antigens to other laboratories; advice on methodology and the interpretation of results; and organization of quality control and research and development within the field of expertise of the individual laboratories.

It has taken time to set up the supraregional service, owing to the need for preparing a detailed explanatory booklet, containers for the transport of samples (either deep-frozen or at ambient temperature), and appropriate request forms. The advantages for clinicians and their patients should outweigh the cost and effort required, if the service has the flexibility to continually adjust to changing needs. Its main role must be the provision of trained staff and of reagents for regional and area centres, so that in time most assays may be undertaken reasonably close to the patient.

\section{Retinitis Pigmentosa}

The eye is a favourite harbour for dystrophic or abiotrophic changes: indeed retinal dystrophies are responsible for about a fifth of all the blindness in Britain. This proportion has been steadily rising as ophthalmologists have become more efficient at dispersing cataracts and at recognizing glaucoma in its early and more arrestible stages.

Most of these diverse dystrophies affect the macular region, and they are largely restricted to the elderly, with only a short life-expectance ahead; and for these patients, though reading may become difficult, other activities are barely curtailed. But the dystrophies such as retinitis pigmentosa (primary pigmentary degeneration), which start peripherally, are much more damaging, since they usually arise in adolescent men and lead to total blindness within a few years.

Retinitis pigmentosa is characterized by deposition throughout the equatorial region of the fundus of patches of fine black pigment, in their shape resembling bone-corpuscles. The condition is hereditary, usually recessive, and most frequent in males (though transmitted, like haemophilia, through the female); and the gene is frequently associated with those of other systemic disorders (for example, polydactyly, obesity, hypogenitalism, and mental retardation-the Laurence-MoonBiedl syndrome). Retinitis pigmentosa is one of the most common of all the genetically-determined eye disorders, affecting about 5 in every 1,000 of the world's population. It is rare in coloured races, and predictably common among inbred island communities, such as those of Tristan da Cunha or of the Scottish islands (where a recent case was traced to a grandfather's incest with his granddaughter). The carrier state can sometimes be recognized by fluorescein angiography and testing rhodospin concentration, and may be confirmed by electrical tests of retinal function-though most female carriers of the X-linked form already show some limited disturbance of the fundus periphery. ${ }^{1}$

Recognition of the heterozygous state in these X-linked forms has become important recently because of the increasing availability of genetic counselling, and the ease of contraception. Antenatal diagnosis is not yet possible, however, since there is no detectable enzymatic abnormality.

Many treatments have been proposed (though such hereditary dystrophies are by their very essence untreatable) and have had ephemeral acclaim when they happened to coincide with a natural remission. Most of these, based on the obviousthough clearly secondary-arterial attentuation, sought to achieve a retinal vasodilatation. The most famous was that of tissue therapy, inaugurated by Filatov in Odessa during the last war, which initially entailed the burial of pieces of placenta within the orbit; then, as the political dichotomy of Europe deepened, wilder and wilder claims came from the subservient Eastern European satellites, announcing the success of an increasing diversity of substances (even vegetable and olive oil) in equally diverse bodily sites, all of which were said to benefit an increasing retinue of diseases (such as myopic degeneration and hypopyon keratitis). However, after the death of Stalin, along with the discrediting of Lysenko, the claims of Filatov's tissue therapy were decently and expeditiously buried. 
Not all cases of retinitis pigmentosa are hereditary, and there are many atypical forms, including those where the clinical changes are limited to one eye, one sector of the fundus, or present as a "retinitis pigmentosa sine pigmento." It has been suggested that this disease may really be little more than a syndrome, and that damage to the visual cells may be provoked by quite disparate causes, since the specialized tissue of the retina can react in only a limited way; and, furthermore, since the antigenicity of the retinal photoreceptors has been generally established, an immunological injury might well be responsible for such degenerative changes.

There have been several recent studies of the biochemical features of retinitis pigmentosa, which generally indicate an increase in the immunoglobulins, ${ }^{2}$ especially IgM, suggesting an infective or autoallergic process. The histological appearances rarely show any evidence of inflammatory reaction, but the activity of slow viruses has been known to provoke macular damage, and such an occult virus infection might well underlie some cases. $\mathrm{Rahi}^{2}$ concluded in a recent survey that the raised IgM level might either cause the retinal degeneration directly or through the intermediary of such an occult (? slow virus) infection, or that individuals with a raised IgM (whose production is genetically controlled) might be more prone to retinal degeneration, or else that the high IgM level might simply represent a further expression of the retinitis pigmentosa genetic anomaly.

\footnotetext{
1 Jay, B., and Bird, A., Transactions of the American Academy of Ophthalmology and Otolaryngology, 1973, 77, 641.

2 Rahi, A. H S., British fournal of Ophthalmology, 1973, 57, 904.
}

\section{Cold and the Heart}

The atmospheric climate of Britain, if not the financial, appears to be improving. Lulled by two relatively mild English winters it may be salutory to remind ourselves of some of the hardships of our cardiac patients in prolonged periods of cold weather. Most doctors are familiar with the problems that a patient's angina pectoris may become worse in cold weather, and stepping out into the cold air first thing on a winter morning may precipitate a sharp attack of chest pain on minimal exercise. Physicians who see large numbers of hypertensive patients have long had the impression that the blood pressure of their patients tends to be higher on cold winter days than in the balmy days of June, and this has recently been confirmed. ${ }^{1}$ The two observations have been linked in a recent report from Portland, Oregon, which suggests that there may be a correlation between exacerbations of angina pectoris and this rise of blood pressure produced by exposure to cold. ${ }^{2}$

Neill et al..$^{2}$ set out to find the mechanism of exacerbation of angina by the cold. In an experiment there are many ways of applying a cold stimulus-locally, diffusely to the skin, or by the inhalation of cold air; but the haemodynamic responses appear to be similar in each instance. ${ }^{3}$ The method chosen in this instance was to place, for either two or six minutes, a damp towel filled with ice chips on the foreheads of 19 patients with coronary heart disease and 6 control patients, all of whom were undergoing left heart catheterization for diagnostic purposes. All consented to the experiments with cold as a separate risk. In the patients exposed to cold for six minutes local discomfort and pain was maximal between one and two minutes of exposure and subsequently decreased. Five patients with ischaemic heart disease (all of whom gave a history of cold intolerance) developed typical angina pectoris after two to three minutes exposure. Parallel with the local discomfort there was a marked increase in both systolic and diastolic blood pressure and a slight increase in heart rate, which was maximal after some six minutes exposure. Coronary blood flow, measured by a xenon disappearance technique, was also increased by the cold and was greater at two minutes than six minutes. As well as changes in systemic arterial pressure cold exposure produced increases in left ventricular end diastolic pressure and left ventricular myocardial oxygen consumption (calculated as the product of coronary blood flow and coronary arteriovenous oxygen difference). All these changes occurred in both groups of patients studied-controls and those with ischaemic heart disease. In seven of the latter exposure to cold resulted in evidence of myocardial hypoxia - an increase in the lactate/pyruvate ratio in coronary venous blood of more than $15 \%$ over the resting level. ${ }^{4}$ These changes suggestive of myocardial hypoxia were not, however, confined to patients with a history of cold intolerance.

A different form of stress to the myocardial oxygen supply -production of a tachycardia by atrial pacing-produced the same evidence of myocardial hypoxia as did cold exposure in the patients with ischaemic heart disease, suggesting that their myocardial hypoxia seems not to be a special response to cold but simply manifestation of diminished coronary reserve.

So it seems that the increase in systemic arterial blood pressure produced by exposure to cold causes an increase in oxygen consumption by the heart. In patients whose coronary circulation is already compromized this extra demand produces myocardial hypoxia and angina pectoris results. This explanation has a better physiological ring to it than previous hypotheses such as "coronary artery spasm," for which there is little factual evidence.

What advice can we give patients with angina pectoris produced by cold exposure? Apart from the rather impractical suggestion of moving to warmer climes, they should be reminded to take their glyceryl trinitrite before stepping out on a cold morning. Further, since $\beta$-blocking drugs help the patient with angina by diminishing myocardial oxygen requirements a seasonal increase in the drug dose might be appropriate.

\footnotetext{
1 Dollery, C. T., British Medical Bulletin, 1973, 29, 158.

2 Neill, W. A., et al., American fournal of Medicine, 1974, 56, 471. 3 Leon, D. F., Amidi, M., and Leonard, J. J., American fournal of Cardi-

${ }^{4}$ Neill, W. A., American fournal of Cardiology, 1968, 22, 507.
}

\section{Squint}

A squint simply means a deviation of the visual axes (not, as people often imagine, eyes that look obliquely, are halfclosed, or inspect at a very close range); but not only is the name confusing-hence the occasional use of that clumsy classicism strabismus-but the subject is so complex that international congresses and frequent symposia have become regular features of the ophthalmological scene.

Such deviations are usually in the horizontal plane and may be convergent or divergent. They may be obvious if 